\title{
Time-Optimal Aircraft Pursuit-Evasion with a Weapon Envelope Constraint - Final Report
}

June, 1990

Research Supported by

NASA Ames-Dryden Flight Research Facility

Grant No. NCC 2-506

Principal Investigator: P. K. A. Menon

NASA Grant Monitor: E. L. Duke

Georgia Institute of Technology

School of Aerospace Engineering

Atlanta, GA 30332

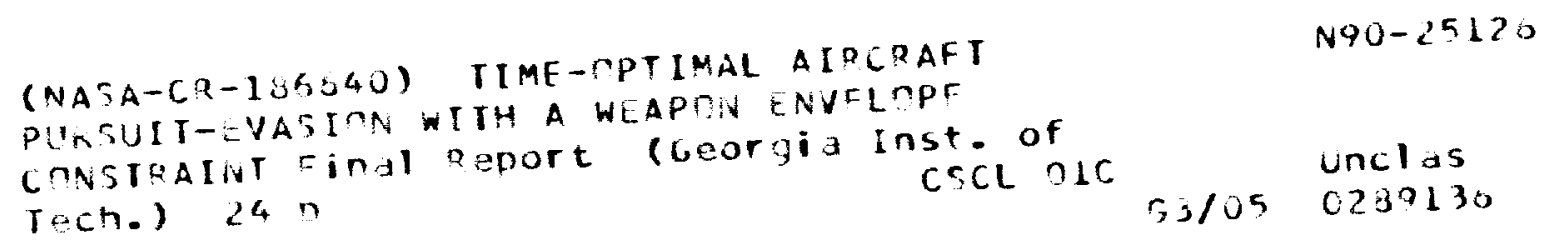




\title{
Time-Optimal Aircraft Pursuit-Evasion with A Weapon Envelope Constraint*
}

\author{
P. K. A. Menon \\ School of Aerospace Engineering \\ Georgia Institute of technology, Atlanta, GA 30332. \\ and \\ E. L. Duke \\ OFDC, NASA Ames-Dryden Flight Research Facility \\ Edwards, CA 93523
}

\begin{abstract}
Optimal pursuit-evasion problem between two aircraft including a realistic weapon envelope is analyzed using differential game theory. This study employs sixth order nonlinear point mass vehicle models and allows the inclusion of an arbitrary weapon envelope geometry. The performance index is a linear combination of flight time and the square of the vehicle acceleration. Closed form Solution to this highorder differential game is then obtained using feedback linearization. The solution is in the form of a feedback guidance law together with a quartic polynomial for time-to-go. Due to its modest computational requirements, this nonlinear guidance law useful for on-board real-time implementation.
\end{abstract}

\section{Introduction}

The objective of this paper is to develop an optimal nonlinear guidance scheme for aircraft pursuitevasion using differential game theory [1] and the theory of feedback linearization $[2,3]$. This work extends previous research [4] to include time-of-flight in the performance index together with a realistic weapon envelope for the pursuing aircraft. Six state nonlinear point mass models of aircraft with lift, bank angle and throttle controls are employed in this work. The weapon envelope considered is an arbitrary three dimensional manifold with its origin at the vehicle center of gravity. This manifold may be specified as a function of the angle between the line-of-sight vector and the vehicle velocity vector, see Figure 1 for details. The distance between the two aircraft is then redefined as the difference between the relative position vector and components of the weapon effectiveness range measured from the pursuer's center of gravity. The pursuer attempts to drive this distance to zero while the evader attempts to make it as large as possible. The pursuit-evasion game terminates the first time instant this distance vector becomes zero. Both vehicles seek to accomplish their objectives in a time-optimal fashion while satisfying the limits on permissible acceleration levels. It is assumed in the present research that the evader has no offensive capabilities. As a result, this analysis includes only the pursuer's weapon envelope. Note that alternate approaches [5-8] may be required in the case where both vehicles have offensive capabilities. Additionally,

*Presented as a Paper at the 1990 American Control Conference, May 23 - 25, San Diego, CA 
unlike Reference 5, it is assumed here that the weapon envelope cannot be oriented independent of the pursuer's velocity vector.

In the past, differential games of this nature could only be handled by linearizing the vehicle dynamics $[9,10]$ or by the use of simplified models $[7,12]$. Differential game solutions obtained using linearized vehicle dynamics is of dubious value since it is not reasonable to define a nominal trajectory. Use of simplified vehicle models, on the other hand, can result in optimistic or pessimistic results depending on the region of validity of these models.

In practical applications, these factors have led to the formulation of such guidance problems as one-sided optimization problems, accurate only for dealing with nonmaneuvering targets. In order to handle actively maneuvering targets, it is necessary to formulate them as differential games. In this case, however, the numerical complexities preclude real-time implementation. Alternate approaches to these problems consist of off-line construction and storage of a field of extremals with real-time interpolation [12].

In Reference 4, it was shown that feedback solutions are feasible for a class of nonlinear differential games arising in aircraft pursuit-evasion. In that work, the performance index was required to be a quadratic form in the distance between two vehicles and the square of the magnitude of their acceleration. In [4], the nonlinear aircraft models were first transformed into a linear time-invariant form. A differential game was then formulated in transformed coordinates and solved. The resulting guidance law was transformed back to the original space to obtain the nonlinear pursuit-evasion guidance law. This research has been subsequently extended to study spacecraft pursuit-evasion and rendezvous problems [13, 14]. Solution to the differential game in this form requires the knowledge of time-to-go. In vehicle models without actuator saturation, this quantity can be computed exactly as outlined in Reference 13. Recently, the solution given in Reference 4 has been evaluated on a realistic simulation of high performance aircraft [15].

The objective of the present research is to extend this methodology to include time-of-flight in the performance index and a realistic weapon envelope in the model for the pursuing aircraft. Note that the results presented here may be adapted to one-sided guidance problems such as those discussed in References 16 and 17.

\section{Nonlinear Models for the Pursuer and the Evader}

The point-mass equations of motion for an aircraft are given by

$$
\begin{gathered}
\dot{V}=\frac{T(h, M, \eta)-D(h, M, L)}{m}-g \sin \gamma \\
\dot{\chi}=\frac{L \sin \phi}{m V \cos \gamma} \\
\dot{\gamma}=\frac{g}{V}\left(\frac{L \cos \phi}{W}-\cos \gamma\right) \\
\dot{x}=V \cos \gamma \cos \chi \\
\dot{y}=V \cos \gamma \sin \chi \\
\dot{h}=V \sin \gamma
\end{gathered}
$$


The salient assumptions in this model are a flat non-rotating earth, thrust along the path and a quiescent atmosphere. In the equations (1)-(6), $V$ is the airspeed, $\gamma$ the flight path angle, $\chi$ heading angle, $T$ the vehicle thrust, $D$ vehicle drag, $L$ the lift, $g$ acceleration due to gravity, $M$ the Mach number, and $m$ is the vehicle mass. Position of the aircraft in an earth-fixed, inertial frame is given by the down-range $x$, cross-range $y$, and altitude $h$. The control variables in this model are the vehicle lift $L$, bank angle $\phi$, and the throttle setting $\eta$. Note that the vehicle thrust is specified as a nonlinear function of altitude, Mach number and the throttle setting.

The aerodynamic drag is calculated using the drag coefficient $C_{D}$, the airspeed $V$, the atmosphere density $\rho$ and the reference area $s$ as

$$
D=C_{D} \rho V^{2} / 2
$$

In (7), the drag coefficient $C_{D}$ is a nonlinear function of the Mach number $M$ and the lift $L$.

The equations of motion for the evader is in the same form as that of the pursuer. However, the thrust and drag characteristics may be different.

\section{The Pursuer's Weapon Envelope}

The present analysis will include a weapon envelope only for the pursuer. The evader is not assumed to have any offensive capabilities. A more complex formulation will be essential if one assumes the existence of offensive capabilities for the evader. In such a differential game, each participant may attempt to maximize the distance between itself and the other vehicle's weapon envelope, while attempting to drive the adversary into its own weapon envelope. Such a formulation may lead to the study of combat games or two-target games[5-8]. The present research will not address these issues. In all that follows, it will be assumed that the roles of each participant in the game is fixed and remains unchanged for the entire duration of the engagement.

As indicated in the foregoing, the evader is not assumed to have any offensive capabilities. As a result, if the pursuer is successful in bringing the evader within its weapon effectiveness range, then capture is said to have occurred. In the present research, pursuer's weapon envelope is assumed to be a three dimensional manifold with its origin located at the vehicle center of gravity. The weapon effectiveness range is defined as the distance between the vehicle center of gravity and the intersection of the three dimensional manifold defining the weapon envelope with the line-of-sight vector. Details are shown in Figure 1. The weapon effectiveness range is assumed to be a function of the angle between the vehicle velocity vector and the line-of-sight vector. Note that this modeling is consistent with the weapon usage envelope in currently operational fixed wing fighter aircraft.

It is assumed here that the weapon envelope cannot be oriented independent of the vehicle velocity vector. In flight vehicles such as combat helicopters and fighter aircraft with precision fuselage pointing capabilities, an alternate assumption might be more appropriate. Clearly, such capabilities provide additional degrees of freedom in controlling the outcome of the differential game.

In a chosen inertial frame, if the pursuer's velocity vector and the differential position vector between the pursuer and the evader are $\left[\dot{x}_{p} \dot{y}_{p} \dot{h}_{p}\right]^{T}$ and $[\Delta x \Delta y \Delta h]^{T}$ respectively, then the angle between the pursuer's velocity vector and the line-of-sight vector may be computed using the expression:

$$
\cos \delta=\frac{\dot{x}_{p} \Delta x+\dot{y}_{p} \Delta y+\dot{h}_{p} \Delta h}{\sqrt{\Delta x^{2}+\Delta y^{2}+\Delta h^{2}} \sqrt{\dot{x}_{p}^{2}+\dot{y}_{p}^{2}+\dot{h}_{p}^{2}}}
$$

In the expression (8), $\Delta h=h_{e}-h_{p}, \Delta x=x_{e}-x_{p}, \Delta y=y_{e}-y_{p}$. The angle $\delta$ is sometimes termed as the line-of-sight angle. The weapon effectiveness range $R_{w}$ can then be specified as a function of the line-of-sight angle as : 


$$
R_{w}=F(\delta)
$$

For example, if the weapon effectiveness envelope were a cone centered at the vehicle center of gravity, the weapon range may be described as follows:

$$
\begin{gathered}
R_{w}=r, \text { if }|\delta| \leq \delta_{\max } \\
R_{w}=0, \text { otherwise }
\end{gathered}
$$

The quantity $r$ is a specified constant. A prolate spheroid with its major axis oriented along the vehicle velocity vector appears to be a more realistic weapon envelope shape. Note that in this case, the weapons will be effective to a certain degree in the tail aspect also. This is consistent with the existing tactical weapon effectiveness envelopes in operational fighter aircraft. Such a weapon envelope is illustrated in Figure 1. In this case, it is possible to write an explicit expression weapon effectiveness range as

$$
R_{w}=\frac{A}{1+B \cos \delta}
$$

In this expression, $A$ and $B$ are two constants specifying the size and shape of the prolate spheroid. These constants may be related to the minimum and maximum weapon ranges $R_{\min }, R_{\max }$ as:

$$
\begin{aligned}
& A=\frac{2 R_{\min } R_{\max }}{R_{\min }+R_{\max }} \\
& B=\frac{R_{\min }-R_{\max }}{R_{\min }+R_{\max }}
\end{aligned}
$$

Note that the present specification of the weapon envelope can include a kill probability distribution given as a function of the weapon range. Further, any alternate shape for the weapon effectiveness envelope can be included in the ensuing analysis. If the orientation of the line-of-sight vector in the given inertial frame is defined using two angles $\theta$ and $\mu$ such that

$$
\begin{gathered}
\theta=\tan ^{-1} \frac{\Delta h}{\sqrt{\Delta x^{2}+\Delta y^{2}}} \\
\mu=\tan ^{-1} \frac{\Delta y}{\Delta x}
\end{gathered}
$$

the components of the weapon effectiveness range can be resolved into three components in the earth fixed frame as

$$
\begin{gathered}
h_{w}=R_{w} \sin \theta \\
x_{w}=R_{w} \cos \theta \cos \mu \\
y_{w}=R_{w} \cos \theta \sin \mu
\end{gathered}
$$

Next, these quantities may be used to redefine the three components of the relative position between the pursuer and evader as :

$$
z_{1}=x_{p}+\epsilon x_{w}-x_{e}
$$




$$
\begin{aligned}
& z_{2}=y_{p}+\epsilon y_{w}-y_{e} \\
& z_{3}=h_{p}+\epsilon h_{w}-h_{e}
\end{aligned}
$$

The variable $\epsilon$ multiplying the weapon envelope components is included in the foregoing to enable the adjustment of the relative weighting between the weapon envelope and the distance between the two vehicles. Such a trade-off is useful while considering the use of different weapon systems in a given pursuit-evasion scenario. From equations (20)-(22), it may be observed that the distance between the two vehicles can be altered either by changing the distance between the vehicle center of gravities or by orienting the weapon envelope. Both these quantities are directly influenced by the vehicle relative position, velocity, and acceleration components. Note that if the weapon envelope was independently orientable, then this relative distance will depend additionally on the orientation parameters. However, in all that follows, it will be assumed that the weapon envelope cannot be oriented independent of the vehicle velocity vector.

Next assume that the quantities $x_{w}, y_{w}, h_{w}$ and their various time derivatives are available from on-board measurements. Examining the geometric relations (19)-(21), it may be observed that these quantities can be computed if the angles $\delta, \theta, \mu$ and their various time derivatives are available. The assumption that these quantities are available from measurements is crucial for including an arbitrary shaped weapon envelope in the derivation of the present guidance law. If these quantities are not available from on-board measurements, then only a spherical weapon envelope shape can be employed in the analysis. This is because of the fact that the second derivative of the line-of-sight angle depends on jerk. The presence of this term introduces difficulties in transforming the vehicle model into linear, time-invariant form as will be apparent in the next section.

An interesting extension of the formulation discussed in this section is the inclusion of a sensor effectiveness envelope in the analysis. In such a pursuit-evasion game, the maneuvers will not only be influenced by weapon envelope considerations, but also by information trade-offs. A familiar example of the control-information mini-maximization is the timed maximum lateral acceleration maneuver employed by fighter aircraft for missile evasion. The objective here is to take advantage of the fact that missile seeker tracking rate as well as the missile maneuvering capabilities are limited. Following the proposed methodology for the inclusion of the weapon envelope, it is possible to include a sensor effectiveness envelope in the present formulation. Additional situations where such constraints as these arise include the guidance of robots in the presence of workspace envelope and actuator constraints. These issues will not be pursued any further in the present paper.

\section{Feedback linearization}

The chief difficulty in obtaining solutions to differential games using realistic flight vehicle models is that these models are highly nonlinear. Moreover, classical linearization approach using Taylor series expansion is invalid in a differential game setting [7], primarily due to the difficulty in defining a nominal trajectory. However, it can be shown[4] that if the differential game between the two vehicles is formulated in terms of the position state variables and their various derivatives, then feedback linearization approach can be used to obtain a nonlinear feedback solution. The primary thrust of the present research is to extend the work in Reference 4 to include the time-of-flight in the performance index and a weapon envelope in the vehicle model. Feedback linearization is then used to transform the vehicle dynamic models into a linear, time-invariant form. The pursuit-evasion game is formulated in terms of 
the transformed states and solved to obtain the guidance law. Inverse transformation of this guidance law to the original coordinates produces an implementable nonlinear guidance scheme.

As in Reference 4, feedback linearization is accomplished by differentiating the equations (20)-(22) twice with respect to time and substituting for $\dot{V}, \dot{\gamma}, \dot{\chi}$ from expressions (1)-(3). interpreting the right hand sides of the resulting expressions as the new control variables in the problem, one has :

$$
\begin{array}{ll}
\dot{z}_{1}=Z_{1}, & \dot{Z}_{1}=U_{1}+\epsilon V_{1}-W_{1} \\
\dot{z}_{2}=Z_{2}, & \dot{Z}_{2}=U_{2}+\epsilon V_{2}-W_{2} \\
\dot{z}_{3}=Z_{3}, & \dot{Z}_{3}=U_{3}+\epsilon V_{3}-W_{3}
\end{array}
$$

where

$$
\begin{gathered}
U_{1}=\frac{\left(T_{p}-D_{p}\right)}{m_{p}} \cos \gamma_{p} \cos \chi_{p}-\frac{L_{p}}{m_{p}}\left(\sin \gamma_{p} \cos \chi_{p} \cos \phi_{p}+\sin \chi_{p} \sin \phi_{p}\right) \\
U_{2}=\frac{\left(T_{p}-D_{p}\right)}{m_{p}} \cos \gamma_{p} \sin \chi_{p}+\frac{L_{p}}{m_{p}}\left(\cos \chi_{p} \sin \phi_{p}-\sin \gamma_{p} \sin \chi_{p} \cos \phi_{p}\right) \\
U_{3}=\frac{\left(T_{p}-D_{p}\right)}{m_{p}} \sin \gamma_{p}+\frac{L_{p}}{m_{p}} \cos \gamma_{p} \cos \phi_{p}-g \\
V_{1}=\ddot{x}_{w}, V_{2}=\ddot{y}_{w}, V_{3}=\ddot{h}_{w} \\
W_{1}=\frac{\left(T_{e}-D_{e}\right)}{m_{e}} \cos \gamma_{e} \cos \chi_{e}-\frac{L_{e}}{m_{e}}\left(\sin \gamma_{e} \cos \chi_{e} \cos \phi_{e}+\sin \chi_{e} \sin \phi_{e}\right) \\
W_{2}=\frac{\left(T_{e}-D_{e}\right)}{m_{e}} \cos \gamma_{e} \sin \chi_{e}+\frac{L_{e}}{m_{e}}\left(\cos \chi_{e} \sin \phi_{e}-\sin \gamma_{e} \sin \chi_{e} \cos \phi_{e}\right) \\
W_{3}=\frac{\left(T_{e}-D_{e}\right)}{m_{e}} \sin \gamma_{e}+\frac{L_{e}}{m_{e}} \cos \gamma_{e} \cos \phi_{e}-g
\end{gathered}
$$

Note that the new control variables $U_{i}, W_{i}, i=1,2,3$ depend on the system states and the original control variables. If the parameter $\epsilon$ is set to zero, the model (23)-(25) will turn out to be identical to that given in Reference 4. It is assumed here that the various derivatives of the weapon envelope components $x_{w}, y_{w}, h_{w}$ are available from on-board measurements. In the general case, these derivatives will depend on the vehicle position, velocity, acceleration, jerk and various time derivatives of jerk. As a result, if these quantities are not available as measurements, the feedback linearization implied by the expressions (23)-(25) will not be feasible. In that case, the ensuing analysis will permit only the use of a spherical weapon envelope.

With the interpretation of $U_{1}+\epsilon V_{1}, U_{2}+\epsilon V_{2}, U_{3}+\epsilon V_{3}, W_{1}, W_{2}, W_{3}$ as the new control variables, the equations (23)-(25) describe a linear time-invariant system. Given the pseudo-control variables, the real control variables in the system can be computed using the relations [4]:

$$
\phi_{p}=\tan ^{-1}\left[\frac{U_{2} \cos \chi_{p}-U_{1} \sin \chi_{p}}{\cos \gamma_{p}\left(U_{3}+g\right)-\sin \gamma_{p}\left(U_{1} \cos \chi_{p}+U_{2} \sin \chi_{p}\right)}\right]
$$




$$
\begin{gathered}
L_{p}=\frac{m_{p}\left[\cos \gamma_{p}\left(U_{3}+g\right)-\sin \gamma_{e}\left(U_{1} \cos \chi_{p}+U_{2} \sin \chi_{p}\right)\right]}{\cos \phi_{p}} \\
T_{p}=\left[\sin \gamma_{p}\left(U_{3}+g\right)+\cos \gamma_{p}\left(U_{1} \cos \chi_{p}+U_{2} \sin \chi_{p}\right)\right] m_{p}+D_{p}
\end{gathered}
$$

The corresponding expressions for the evader's control variables can be obtained by replacing $U_{1}, U_{2}, U_{3}$ by $W_{1}, W_{2}, W_{3}$ and changing the state variable subscripts as follows :

$$
\begin{gathered}
\phi_{e}=\tan ^{-1}\left[\frac{W_{2} \cos \chi_{e}-W_{1} \sin \chi_{e}}{\cos \gamma_{e}\left(W_{3}+g\right)-\sin \gamma_{e}\left(W_{1} \cos \chi_{e}+W_{2} \sin \chi_{e}\right)}\right] \\
L_{e}=\frac{m_{e}\left[\cos \gamma_{e}\left(W_{3}+g\right)-\sin \gamma_{e}\left(W_{1} \cos \chi_{e}+W_{2} \sin \chi_{e}\right)\right]}{\cos \phi_{e}} \\
T_{e}=\left[\sin \gamma_{e}\left(W_{3}+g\right)+\cos \gamma_{e}\left(W_{1} \cos \chi_{e}+W_{2} \sin \chi_{e}\right)\right] m_{e}+D_{e}
\end{gathered}
$$

Physically, the pseudo-control variables $U_{i}, W_{i}, i=1,2,3$ are the acceleration components of the vehicle in the chosen inertial frame. Since the magnitude of a vector is invariant under coordinate transformation, the magnitudes of $U, W$ are also the pursuer-evader acceleration magnitudes in the flight path axis system.

The attitude dynamics of the pursuer and the evader were not included in the foregoing analysis. Note that these may be included at the expense of increased model complexity. The primary reason for including these in a differential game would be to study the various information trade-offs involved in a typical pursuit-evasion scenario. For instance, if the pursuer's weapons employed an active radar seeker, then its maneuvers would be influenced by the fact that the maximum target area must be visible at all times. The evader, on the other hand, would employ an opposite strategy. Several interesting variations of this differential game can be studied in the present setting.

\section{Guidance Law for Aircraft Pursuit-Evasion}

The previous section dealt with an approach for making the nonlinear aircraft models amenable to analysis. In this section, the pursuit-evasion differential game will be formulated in the transformed coordinates and solved. Inverse transformation of this solution to the original coordinates yields the nonlinear feedback guidance law. In the following development it will be assumed that all the state variables required for computing the feedback law are known perfectly. Once the differential game is solved with perfect information, the effects of incomplete or imperfect information can be investigated using this solution.

The first issue in differential games is that of role definition. There is a controversy on this issue currently. But if the roles are assigned at the outset, the resulting differential game is amenable to analysis via Isaac's theory [1]. Assuming that the roles have been defined, the objective of the pursuer is to minimize the specified performance index which the evader tries to maximize. The performance index employed in the present research is:

$$
\begin{gathered}
\min _{(U+\epsilon V)} \max _{W} \int_{0}^{t_{f}}\left\{\zeta+\frac{(1-\zeta)}{2}\left[a\left[\left(U_{1}+\epsilon V_{1}\right)^{2}+\left(U_{2}+\epsilon V_{2}\right)^{2}+\left(U_{3}+\epsilon V_{3}\right)^{2}\right]\right.\right. \\
\left.\left.-b\left[W_{1}{ }^{2}+W_{2}^{2}+W_{3}{ }^{2}\right]\right]\right\} d t
\end{gathered}
$$


The final time $t_{f}$ is unspecified. This performance index is to be optimized by the two participants subject to the differential constraints (23)-(25). In (39), $\zeta$ defines the relative weighting between flight time and acceleration magnitude, while the positive quantities $a$ and $b$ serve to constrain the acceleration magnitudes of the pursuer and the evader. For reasons that will be made clear in the ensuing, it is assumed that

$$
b>a, 0<\zeta<1
$$

The negative sign in front of the evader's acceleration term explicitly identifies this player as the maximizer. Initial values on all the state variables are assumed known. The pursuit-evasion maneuvers are terminated the first time instant the evader makes contact with the pursuer's weapon envelope, viz.

$$
z_{1 f}, z_{2 f}, z_{3 f}=0
$$

where

$$
z_{1 f}=z_{1}\left(t_{f}\right), z_{2 f}=z_{2}\left(t_{f}\right), z_{3 f}=z_{3}\left(t_{f}\right)
$$

The final value of the relative velocities $Z_{1}, Z_{2}, Z_{3}$ are assumed to be free. Next, define the variational Hamiltonian as:

$$
\begin{aligned}
H= & \zeta+\frac{1}{2}(1-\zeta)\left\{a\left[\left(U_{1}+\epsilon V_{1}\right)^{2}+\left(U_{2}+\epsilon V_{1}\right)^{2}+\left(U_{3}+\epsilon V_{1}\right)^{2}\right]-b\left[W_{1}^{2}+W_{2}^{2}+W_{3}^{2}\right]\right\} \\
& +\lambda_{1} Z_{1}+\lambda_{2} Z_{2}+\lambda_{3} Z_{3}+\lambda_{4}\left(U_{1}+\epsilon V_{1}-W_{1}\right)+\lambda_{5}\left(U_{2}+\epsilon V_{2}-W_{2}\right)+\lambda_{6}\left(U_{3}+\epsilon V_{3}-W_{3}\right)
\end{aligned}
$$

As in Reference 4, the objective of the present research is obtain a saddle-point solution to the differential game. The conditions under which such a solution may exist are well known [18]. The central requirement here is the separability of the variational Hamiltonian with respect to the pursuer and the evader state and control variables. In the present case, inspection of the variational Hamiltonian defined in (38) will reveal that such a separability exists. The saddle point solution can be found by proceeding formally as follows.

The costate equations [10] for this problem can be obtained as

$$
\begin{gathered}
\dot{\lambda}_{1}=\dot{\lambda}_{2}=\dot{\lambda}_{3}=0 \\
\dot{\lambda}_{4}=-\lambda_{1}, \dot{\lambda}_{5}=-\lambda_{2}, \dot{\lambda}_{6}=-\lambda_{3}
\end{gathered}
$$

The optimality conditions yield :

$$
\begin{aligned}
& U_{1}+\epsilon V_{1}=\frac{-\lambda_{4}}{a(1-\zeta)} \\
& U_{2}+\epsilon V_{2}=\frac{-\lambda_{5}}{a(1-\zeta)} \\
& U_{3}+\epsilon V_{3}=\frac{-\lambda_{6}}{a(1-\zeta)}
\end{aligned}
$$




$$
\begin{aligned}
& W_{1}=\frac{-\lambda_{4}}{b(1-\zeta)} \\
& W_{2}=\frac{-\lambda_{5}}{b(1-\zeta)} \\
& W_{3}=\frac{-\lambda_{6}}{b(1-\zeta)}
\end{aligned}
$$

Since the final values of $Z_{1}, Z_{2}, Z_{3}$ are free, the costates $\lambda_{4}, \lambda_{5}, \lambda_{8}$ are zero at the final time. This fact, together with equations (45)-(50) imply that the pseudo control variables are all zero at the final time. Integrating the costate equations (44) and using the boundary conditions on $\lambda_{4}, \lambda_{5}, \lambda_{6}$ yields

$$
\begin{aligned}
& \lambda_{4}=\lambda_{1}\left(t_{f}-t\right) \\
& \lambda_{5}=\lambda_{2}\left(t_{f}-t\right) \\
& \lambda_{6}=\lambda_{3}\left(t_{f}-t\right)
\end{aligned}
$$

Using the expressions (51) - (53) to eliminate the costates on the right hand side of the optimality conditions $(45)-(50)$ one has :

$$
\begin{aligned}
& \dot{Z}_{1}=\frac{\lambda_{1}}{(1-\zeta)}\left[\frac{1}{b}-\frac{1}{a}\right]\left(t_{f}-t\right) \\
& \dot{Z}_{2}=\frac{\lambda_{2}}{(1-\zeta)}\left[\frac{1}{b}-\frac{1}{a}\right]\left(t_{f}-t\right) \\
& \dot{Z}_{3}=\frac{\lambda_{3}}{(1-\zeta)}\left[\frac{1}{b}-\frac{1}{a}\right]\left(t_{f}-t\right)
\end{aligned}
$$

Expressions (54)-(56) may next be integrated to obtain $Z_{1}, Z_{2}, Z_{3}$. Due to the symmetry of the solution to this problem, only one component of the solution will be fully illustrated in the ensuing. Thus :

$$
Z_{1}=Z_{1}(0)+\frac{\lambda_{1}}{(1-\zeta)}\left[\frac{1}{b}-\frac{1}{a}\right]\left(t_{f} t-\frac{t^{2}}{2}\right)
$$

Integrating the expression (57) yields

$$
z_{1}(t)=z_{1}(0)+Z_{1}(0) t+\frac{\lambda_{1}}{(1-\zeta)}\left[\frac{1}{b}-\frac{1}{a}\right]\left(t_{f} \frac{t^{2}}{2}-\frac{t^{3}}{6}\right)
$$

Using the game termination condition (41) in the expression (58) yields

$$
\lambda_{1}=-\frac{3(1-\zeta) a b}{(a-b) t_{f}^{3}}\left[z_{1}(0)+Z_{1}(0) t_{f}\right]
$$

This may next be substituted in the optimality conditions to obtain the optimal control for the pursuer as

$$
U_{1}+\epsilon V_{1}=\frac{3 b}{(a-b) t_{f}^{3}}\left[z_{1}(0)+Z_{1}(0) t_{f}\right]\left(t_{f}-t\right)
$$


The optimal control for the evader is given by

$$
W_{1}=\frac{3 a}{(a-b) t_{f}^{3}}\left[z_{1}(0)+Z_{1}(0) t_{f}\right]\left(t_{f}-t\right)
$$

The remaining control variables in the problem can be similarly computed. The solution is incomplete at this stage since the final time $t_{f}$ is unknown. This quantity may be computed by invoking a constant of motion in this problem. Since the final time is open and the variational Hamiltonian is autonomous, one has that

$$
H(t)=0
$$

Substituting for the optimal controls in terms of costates in the constant of motion (62) at the initial time results in

$$
\begin{array}{r}
0=\zeta+\frac{1}{2(1-\zeta)}\left[\frac{1}{b}-\frac{1}{a}\right]\left\{\lambda_{4}{ }^{2}+\lambda_{5}{ }^{2}+\lambda_{6}{ }^{2}\right\} \\
+\lambda_{1} Z_{1}(0)+\lambda_{2} Z_{2}(0)+\lambda_{3} Z_{3}(0)
\end{array}
$$

Next, substituting for $\lambda_{4}, \lambda_{5}, \lambda_{6}$ from expressions (51) - (53) yields

$$
\begin{array}{r}
0=\zeta+\frac{1}{2(1-\zeta)}\left[\frac{1}{b}-\frac{1}{a}\right]\left\{\lambda_{1}{ }^{2}+\lambda_{2}{ }^{2}+\lambda_{3}{ }^{2}\right\} t_{f}{ }^{2} \\
+\lambda_{1} Z_{1}(0)+\lambda_{2} Z_{2}(0)+\lambda_{3} Z_{3}(0)
\end{array}
$$

Finally, substituting for $\lambda_{1}, \lambda_{2}, \lambda_{3}$ in terms of initial states from expression (59) yields a quartic polynomial of the form

$$
t_{f}^{4}+q_{2} t_{f}^{2}+q_{1} t_{f}+q_{0}=0
$$

where

$$
\begin{gathered}
q_{2}=\left[Z_{1}(0)^{2}+Z_{2}(0)^{2}+Z_{3}(0)^{2}\right] / \frac{2 \zeta}{3(1-\zeta)}\left[\frac{1}{b}-\frac{1}{a}\right] \\
q_{1}=4\left[Z_{1}(0) z_{1}(0)+Z_{2}(0) z_{2}(0)+Z_{3}(0) z_{3}(0)\right] / \frac{2 \zeta}{3(1-\zeta)}\left[\frac{1}{b}-\frac{1}{a}\right] \\
q_{0}=3\left[z_{1}(0)^{2}+z_{2}(0)^{2}+z_{3}(0)^{2}\right] / \frac{2 \zeta}{3(1-\zeta)}\left[\frac{1}{b}-\frac{1}{a}\right]
\end{gathered}
$$

The smallest positive value of the final time emerging from the polynomial (65) should be used in subsequent calculations. Note that the parameter $\zeta$ should satisfy the in equality $(40)$ to ensure that these polynomial coefficients remain finite. Additionally, the polynomial coefficients $q_{0}$ and $q_{2}$ will be negative if $b>a$. Since the coefficient corresponding to $t_{f}^{3}$ is zero and the coefficients $q_{0}, q_{2}$ are negative, the Hurwitz criterion [19] in the theory of polynomials implies that this polynomial has roots with positive real parts. Next, forming the Routh array [19], the first column turns out to be

$$
\left[1, \kappa,-q_{1} / \kappa, q_{1}, q_{0}\right]^{T}
$$

Here, $\kappa$ is a small positive parameter and the superscript $T$ denotes the transpose. If $q_{1}$ is less than zero, this array suggests that one root of the polynomial (65) will have a positive real part. On the other 
hand, if $q_{1}>0$, three roots of this polynomial will have positive real parts: Since complex roots always occur in conjugate pairs, the foregoing observations imply that the polynomial (65) will produce at least one usable value of $t_{f}$. Note that the final time $t_{f}$ has to be iteratively determined. However, since the objective is to determine the smallest positive real root of this polynomial, a one dimensional search scheme is adequate.

An interesting special case occurs if $q_{1}$ were zero. Note that the numerator of the coefficient $q_{1}$ is simply the inner product of relative position and velocity vectors at the initial time. If these two vectors are orthogonal, the coefficient $q_{1}$ will be zero. This condition can be seen to be satisfied in various commonly encountered engagement scenarios, one of them being the case of pursuer and evader being instantaneously located at the same down-range-cross-range positions while in level flight at different altitudes and airspeeds. In these cases, the quartic (65) can be solved for in closed form. All the roots of (65) may be computed using the expression

$$
t_{g o}^{2}=\frac{-q_{2} \pm \sqrt{q_{2}^{2}-4 q_{0}}}{2}
$$

Both $q_{2}$ and $q_{0}$ will be negative if $b>a$. In this case, the right hand side of $(70)$ will be strictly real.

Finally, in order to convert the control laws (56), (57) to explicit feedback form, assume that that the current time $t$ is the initial time. In this case, the quantities $\left(t_{f}-t\right), t_{f}$ may both be replaced by time-to-go $t_{g o}$. Additionally, as discussed elsewhere in this paper, the weapon envelope components $V_{1}, V_{2}, V_{3}$ are assumed to be available from measurements. Using these, one has

$$
\begin{gathered}
U_{1}=\frac{3 b}{(a-b) t_{g \circ}^{2}}\left[z_{1}+Z_{1} t_{g \circ}\right]-\epsilon V_{1} \\
U_{2}=\frac{3 b}{(a-b) t_{g \circ}^{2}}\left[z_{2}+Z_{2} t_{g \circ}\right]-\epsilon V_{2} \\
U_{3}=\frac{3 b}{(a-b) t_{g o}^{2}}\left[z_{3}+Z_{3} t_{g \circ}\right]-\epsilon V_{3} \\
W_{1}=\frac{3 a}{(a-b) t_{g o}^{2}}\left[z_{1}+Z_{1} t_{g \circ}\right] \\
W_{2}=\frac{3 a}{(a-b) t_{g o}^{2}}\left[z_{2}+Z_{2} t_{g \circ}\right] \\
W_{3}=\frac{3 a}{(a-b) t_{g o}^{2}}\left[z_{3}+Z_{3} t_{g o}\right]
\end{gathered}
$$

This completes the solution of the differential game in the feedback linearized coordinates. However, this solution is usable only after transformation to the original coordinates. This transformation may be achieved by substituting the expressions (71)-(76) in the expressions (33)-(38) given in the previous section. However, in order to conserve space, this step will not be carried out here.

The guidance laws resulting from the foregoing algebraic manipulations are highly nonlinear and coupled. They use full state feedback along with vehicle performance related quantities such as thrust, drag, and mass for generating the minimax optimal feedback control settings for the pursuer and the evader. It is not difficult to show that these solutions satisfy the Strengthened Legendre-Clebsch necessary condition if $a>0, b>0$. Investigation of additional second-order necessary conditions and a detailed examination of the saddle point properties of this solution will be of future interest. 


\section{Numerical Evaluation}

The nonlinear guidance law developed in this paper was implemented on a point-mass simulation of two aircraft. The vehicle data used for both aircraft is similar that of a high performance aircraft used in previous aircraft pursuit-evasion studies [4,15]. The equations of motion were integrated using a fourth-order Runge-Kutta method and the aerodynamic coefficients and the thrust limits were linearly interpolated from a stored table. The time-to-go quartic (65) was solved using the Newton's method with zero as the initial guess. The derivatives of the weapon envelope components required in the guidance law computations were obtained using a second-order linear observer of the form

$$
\begin{aligned}
& \tilde{x}_{w 0}=\omega^{2}\left(x_{w}-x_{w 0}\right)-2 \nu \omega \dot{x}_{w 0} \\
& \ddot{y}_{w 0}=\omega^{2}\left(y_{w}-y_{w o}\right)-2 \nu \omega \dot{y}_{w 0} \\
& \tilde{h}_{w 0}=\omega^{2}\left(h_{w}-h_{w o}\right)-2 \nu \omega \dot{h}_{w 0}
\end{aligned}
$$

In (77)-(79), $\omega, \nu$ are the observer natural frequency and the damping ratio, respectively. The values used in the present numerical study are $\omega=10, \nu=1$. This observer uses the weapon envelope components $x_{w}, y_{w}, h_{w}$ as the inputs to form the derivative estimates $\dot{x}_{w o}, \dot{y}_{w o}, \dot{h}_{w o}, \ddot{x}_{w o}, \ddot{y}_{w o}, \ddot{h}_{w o}$. These estimates are then used in computing the controls for the pursuer and the evader.

Although several runs have been made, results from one engagement scenario will only be presented in the ensuing. In this study, a highly eccentric prolate spheroid weapon envelope with its major axis aligned along the pursuer's velocity vector was considered. This weapon envelope had a minimum range of $10 \mathrm{~m}$ and a maximum range of $500 \mathrm{~m}$. The two vehicles are initially separated by $2000 \mathrm{~m}$ in down range and $5000 \mathrm{~m}$ in cross range, with the pursuer behind the evader. The pursuer's velocity vector is initially aligned along the down range direction, while the evader's velocity vector points along the cross range direction at the initial time. The pursuer has an initial velocity is $160 \mathrm{~m} / \mathrm{s}$ while flying level at 16 $\mathrm{km}$ altitude. The evader has an initial velocity of $110 \mathrm{~m} / \mathrm{s}$ at an altitude of $10 \mathrm{~km}$, with zero flight path angle. The present study employed a weighting factor of $a=0.01$ for the pursuer and $b=0.03$ for the evader. The weight on the flight time was $\zeta=0.5$. The engagement time corresponding to these initial conditions and the given weights computed from the quartic (65) turns out to be 50.79 seconds. The weapon envelope weighting factor $\epsilon=1$ was used in this analysis.

Figure 2 illustrates the trajectories of both the pursuer and the evader in the cross-range down-range plot. Triangular markers are provided every 5 seconds to give an idea about the relative position of the two vehicles. To further aid in interpreting these trajectories, the flight time is indicated at 10 second intervals along the pursuer-evader trajectories. The turn-dash behavior of both the pursuer and the evader noted in previous studies $[4,12,15]$ is apparent from this figure. The altitude histories corresponding to this engagement are given in Figure 3. Both the pursuer and the evader have negative flight path angles at the time of capture. The heading angle histories for both vehicles are given in Figure 4 . From this figure, it is clear that the pursuer is attempting to continuously avoid a heading angle match. The airspeed histories for the two vehicles given in Figure 5 shows the pursuer slowing down during the initial portion of the turn followed by an acceleration. The evader, on the other hand, is accelerating through most of the engagement. The load factor, throttle setting and the bank angle for both the pursuer and the evader are shown in Figures 6, 7, 8. From these figures, it may be observed that both the pursuer and the evader are executing a descending turn. In order to illustrate the influence of the weapon envelope on the engagement, a plot of the acceleration history along the altitude direction is given in Figure 9 . If the weapon envelope were spherical, the acceleration history would have been a straight line, as is the case 
for the evader. However, the presence of the prolate spheroid envelope introduces strong nonlinearities in the acceleration history. The performance of the pursuit-evasion guidance law is apparent from these plots. Evaluation of these guidance laws in a more complex vehicle simulation is currently under way.

\section{Conclusions}

This paper presented the development of feedback guidance laws for aircraft pursuit-evasion. The analysis employed nonlinear point-mass models of aircraft. A realistic weapon effectiveness envelope was included in the analysis. Assuming that the weapon envelope components may be computed from given measurements, the vehicle model was transformed to a linear, time invariant form. The pursuit-evasion differential game was formulated using this model and the solution obtained. The performance index employed consisted of a linear combination of flight time and the square of the vehicle acceleration. Inverse transformation of this solution produces a nonlinear guidance law together with a quartic for the computation of the free final time. This guidance law is in closed-loop state feedback form and uses the vehicle performance data. Modifications of the present guidance law to include a sensor effectiveness envelope were sketched.

Numerical results using high performance aircraft data were given. Since the computational requirements for the guidance law are modest, it appears that this solution is implementable on-board aircraft.

\section{References}

[1] Isaacs, R., Differential Games, Robert E. Krieger, Huntington, New York, 1965.

[2] Brockett, R. W., "Nonlinear Systems and Differential Geometry", Proceedings of the IEEE, Vol. 64, Feb. 1976, pp. 61-72.

[3] Hunt, L. R., Su, R., and Meyer, G., "Global Transformations of Nonlinear Systems", IEEE Transactions on Automatic Control, Vol. , Jan. 1983, pp. 24-31.

[4] Menon, P. K. A., "Short-Range Nonlinear Feedback Strategies for Aircraft Pursuit-Evasion", Journal of Guidance, Control and Dynamics, Jan.-Feb. 1989, pp. 27-32.

[5] Heymann, M., Ardema, M. D., and Rajan, N., "A Formulation and Analysis of Combat Games", NASA TM 85927, April 1984.

[6] Getz, W. M., and Pachter, M., "Capturability in a Two-Target Game of Two Cars", Journal of Guidance and Control, Vol. 4, Jan.- Feb. 1981, pp.15-21.

[7] Davidovitz, A., and Shinar, J., "Eccentric Two-Target Model for Qualitative Air Combat Game Analysis", Journal of Guidance, Control, and Dynamics, Vol. 8, May-June 1985, pp. 325-331.

[8] Ghose, G., and Prasad, U. R., "Analysis of Security Strategies for a two Target Game", AIAA Guidance, Navigation and Control Conference, Aug. 14-16, 1989, Boston, MA, paper 89-3597.

[9] Anderson, G. M., "Comparison of Air-to-Air Missile Guidance Laws based on Optimal Control and Differential Game Theory", Proceedings of the AIAA Guidance, and Control Conference, August 1979, Paper No. 79-1736.

[10] Bryson, A. E., and Ho, Y. C., Applied Optimal Control, Hemisphere, Washington, 1975. 
[11] Kelley, H. J., and Lefton, L., "Estimation of Weapon-Radius Vs Maneuverability Tradeoff for Airto-Air Combat", AIAA Journal, Vol. 15, Feb. 1977, pp. 145-148.

[12] Ardema, M. D., Rajan, N., and Yang, L., "Three-Dimensional Energy-State Extremals in Feedback Form", Journal of Guidance, Control and Dynamics, July-Aug. 1989, pp. 601-605.

[13] Menon, P. K. A., and Calise, A. J., "Interception, Evasion, Rendezvous, and Velocity-to-be-Gained Guidance for Spacecraft", AIAA Guidance, Navigation, and Control Conference, Monterey, CA., Aug. 1987.

[14] Menon, P. K. A., Calise, A. J., and Leung, S. K. M., "Guidance Laws for Spacecraft Pursuit-Evasion and Rendezvous", AIAA Guidance, Navigation, and Control Conference, Minneapolis, MN, Aug. 1988.

[15] Williams, P. S., Antoniewicz, R. F., Duke, E. L., and Menon, P. K. A., "Study of a Pursuit-Evasion Guidance law for High Performance Aircraft", 1989 American Control Conference, Pittsburgh, PA, June 21-23, 1989.

[16] Calise, A. J., "Singular perturbation Techniques for On-line Optimal Flight Path Control", Journal of Guidance and Control, Vol. 4, July-Aug. 1981, pp. 398-405.

[17] Menon, P. K. A., and Briggs, M. M., "Near-Optimal Midcourse Guidance for Air-to-Air Missiles", to appear in the Journal of Guidance, Control and Dynamics.

[18] Friedmann, A., Differential Games, Academic Press, New York, 1971.

[19] Kuo, B. C., Automatic Control Systems, Prentice-Hall, New York, 1967. 


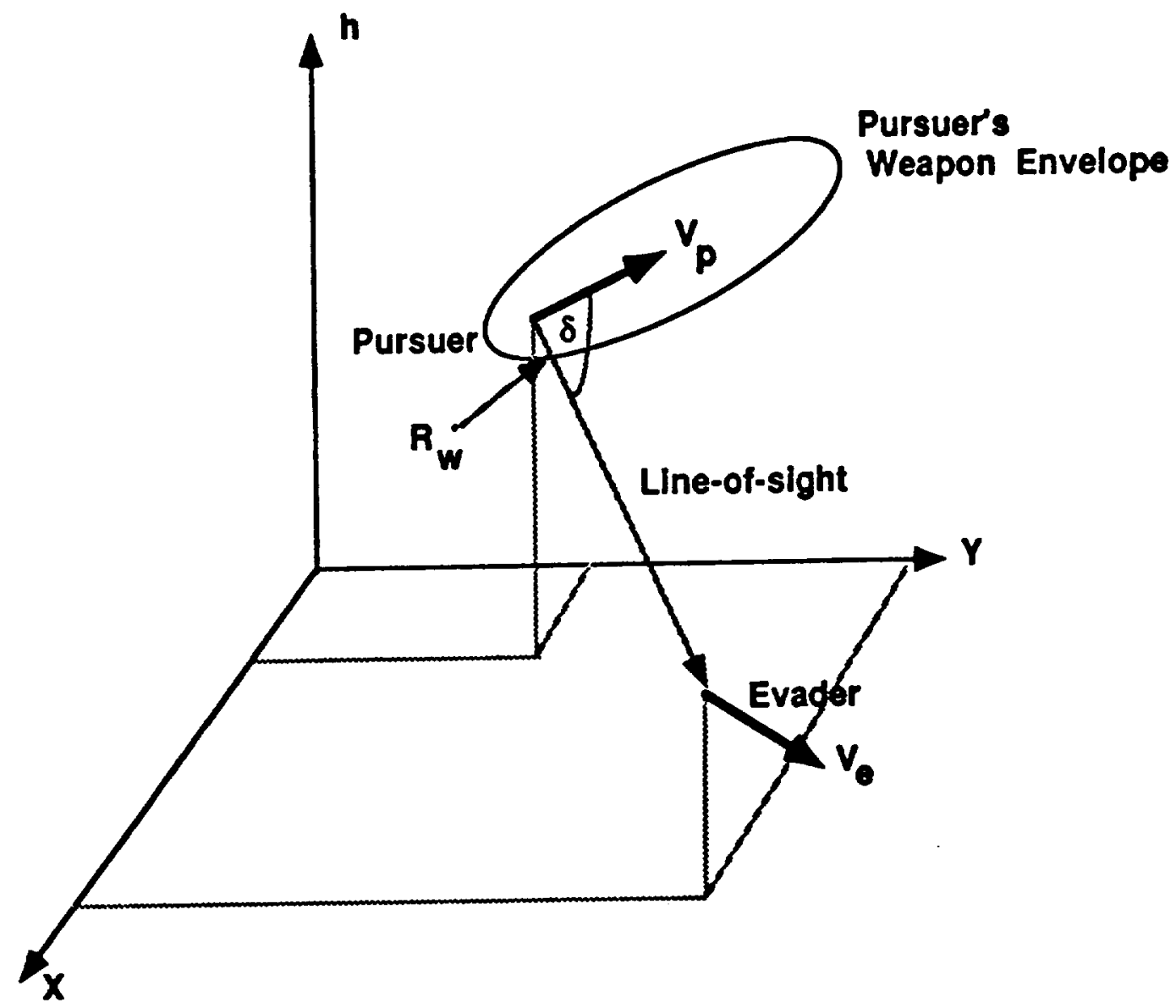

Fig. 1. The Coordinate System 


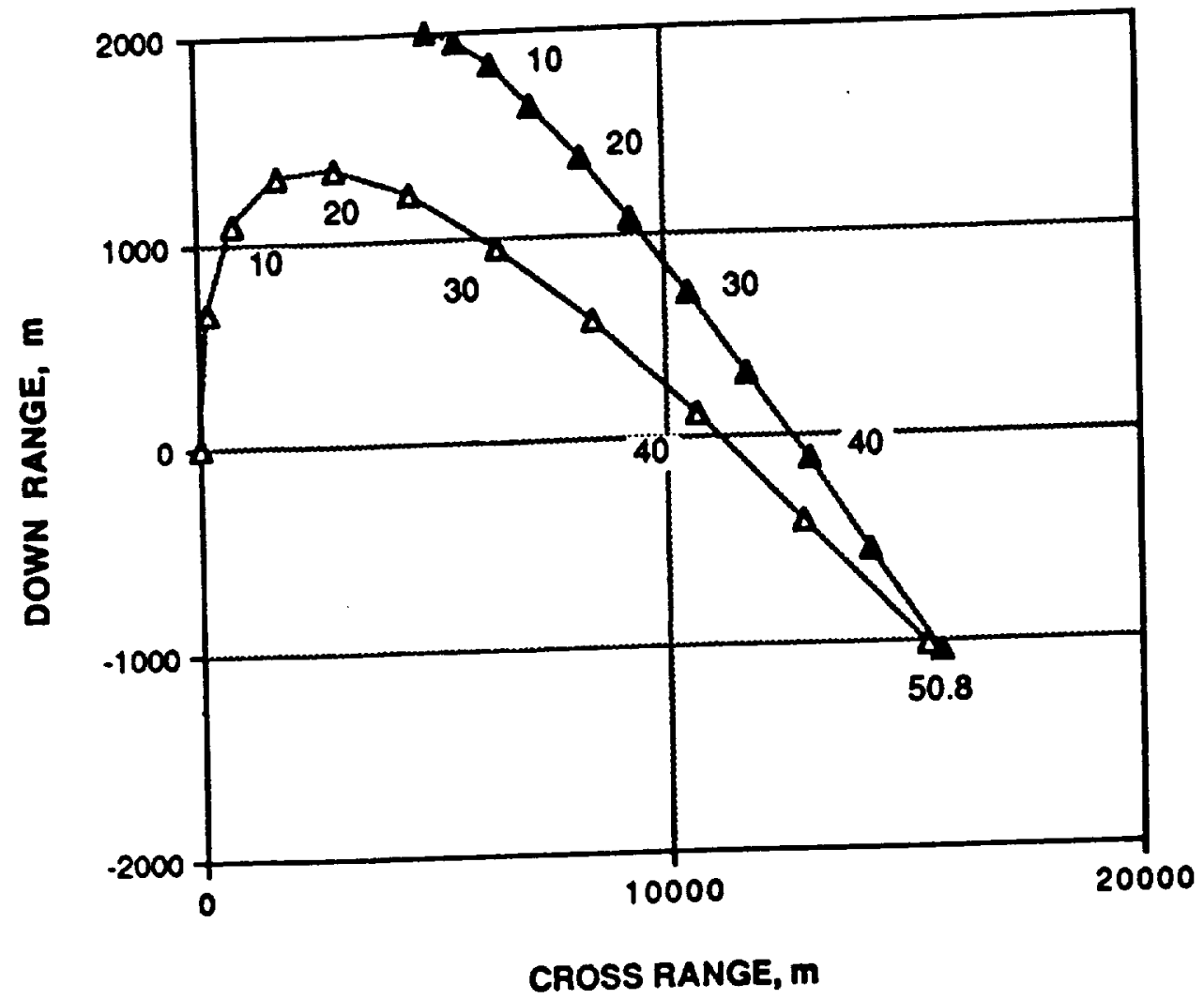

Fig. 2. Pursuer-Evader Trajectorles in the Horizontal Plane. Dark Trlangle : Evader, Light Triangle : Pursuer. 


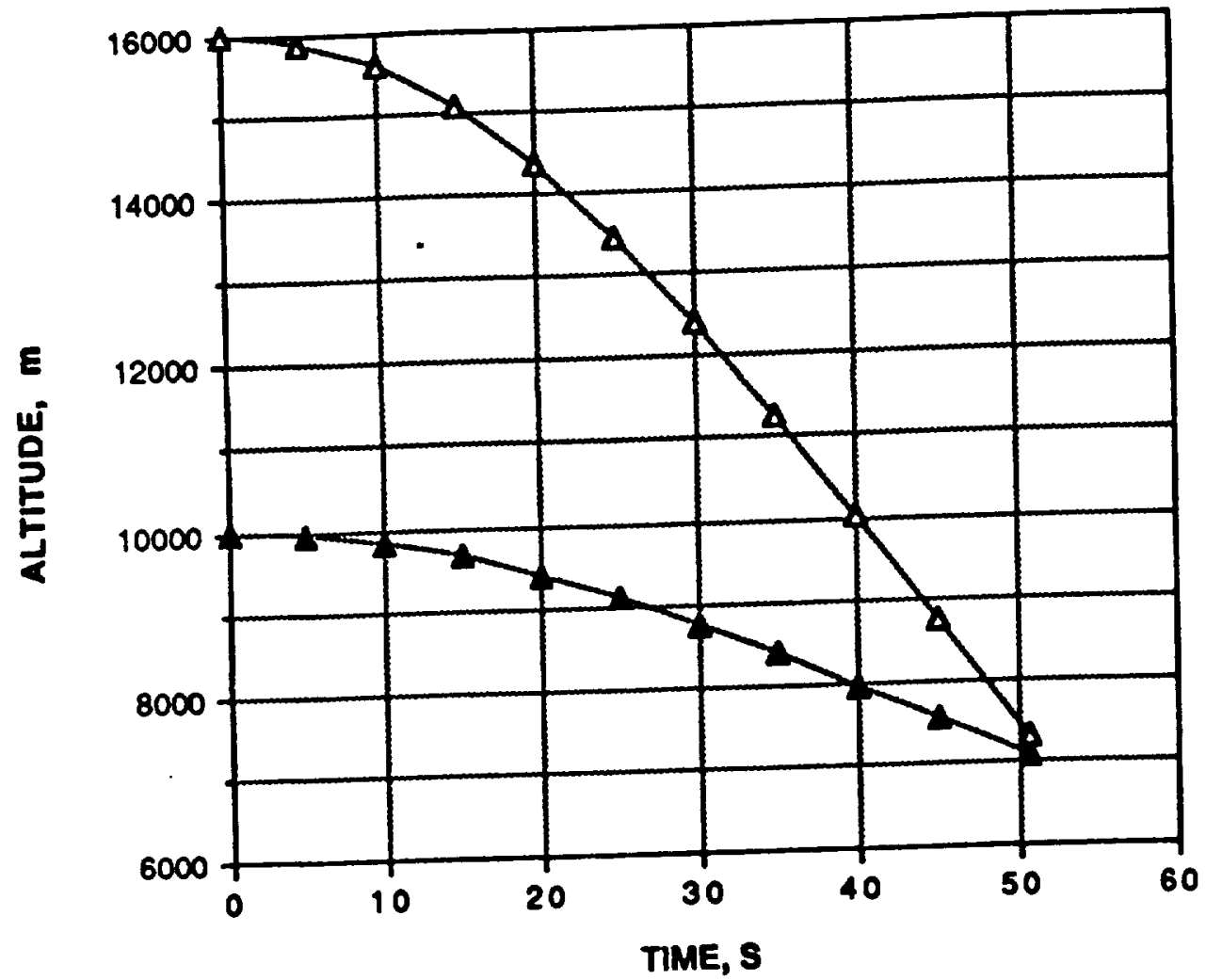
Fig. 3. Temporal Evolution of Pursuer-Evader Altitudes.
Dark Trlangle : Evader, Light Trlangle : Pursuer. 


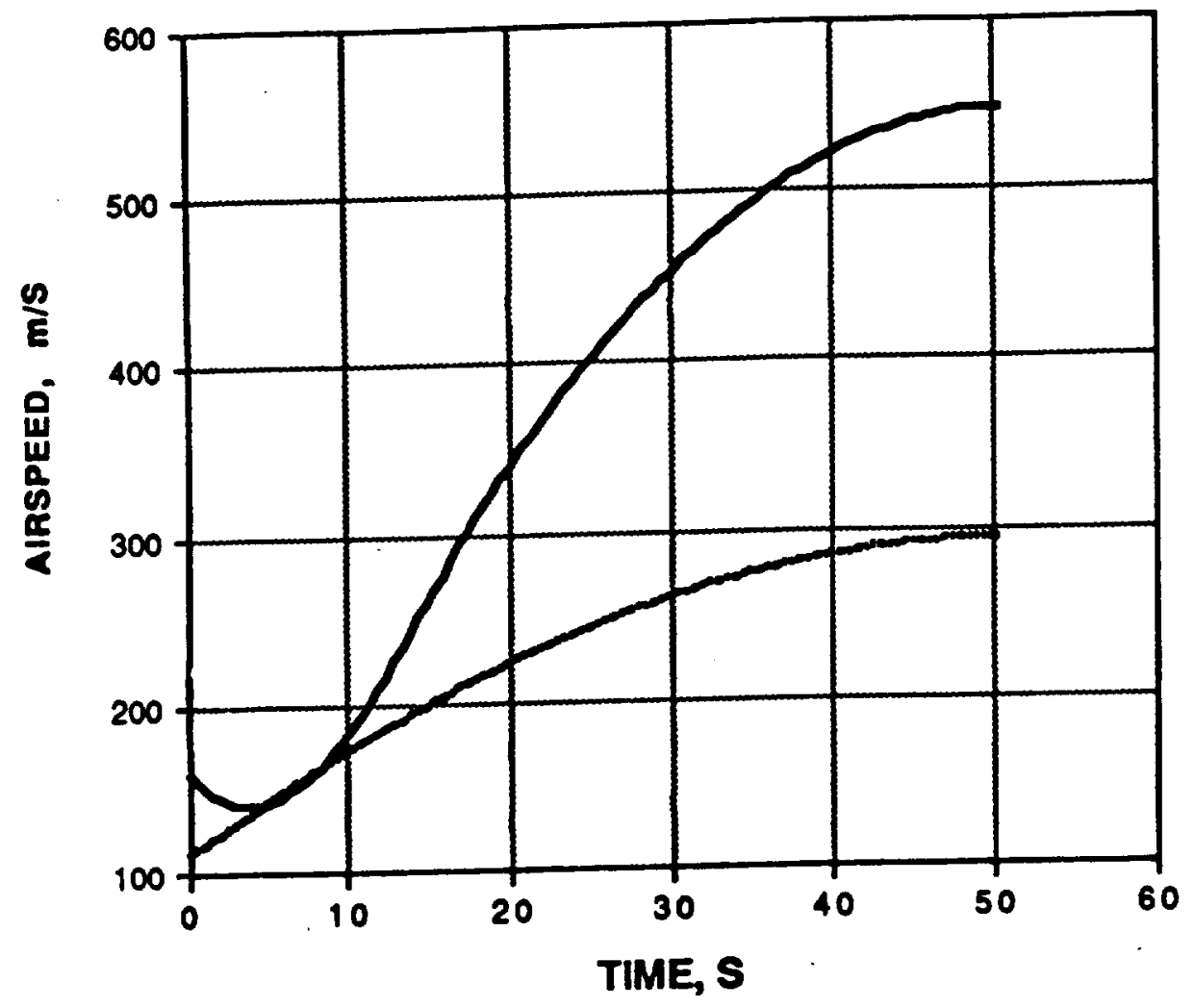

Fig. 4. Temporal Evolution of Pursuer-Evader Alrspeeds. Dotted LIne : Evader, Solld LIne : Pursuer. 


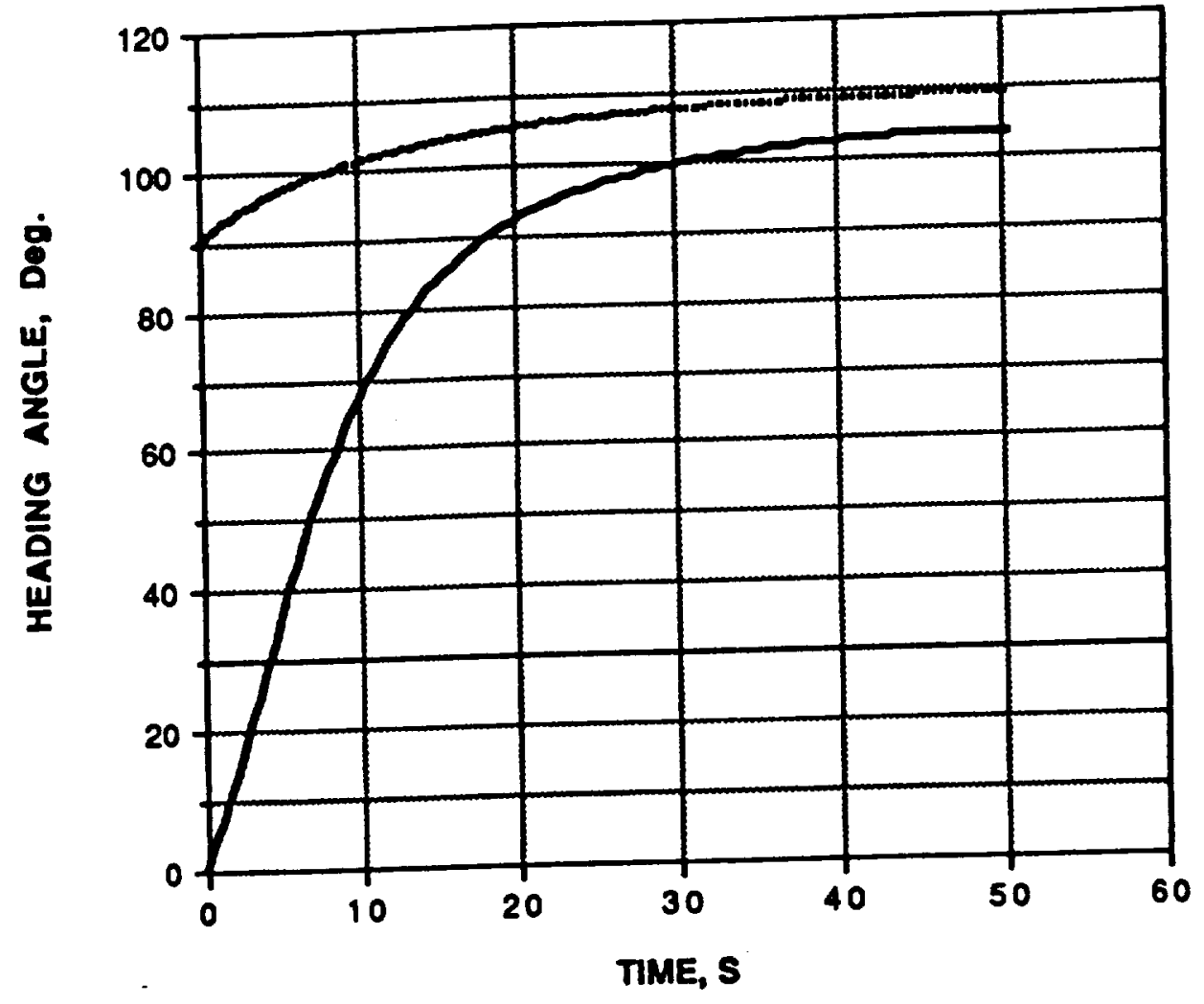

Fig. 5. Heading Angle Histories for the Pursuer and the Evader. Dotted Line : Evader, Solld Line : Pursuer. 


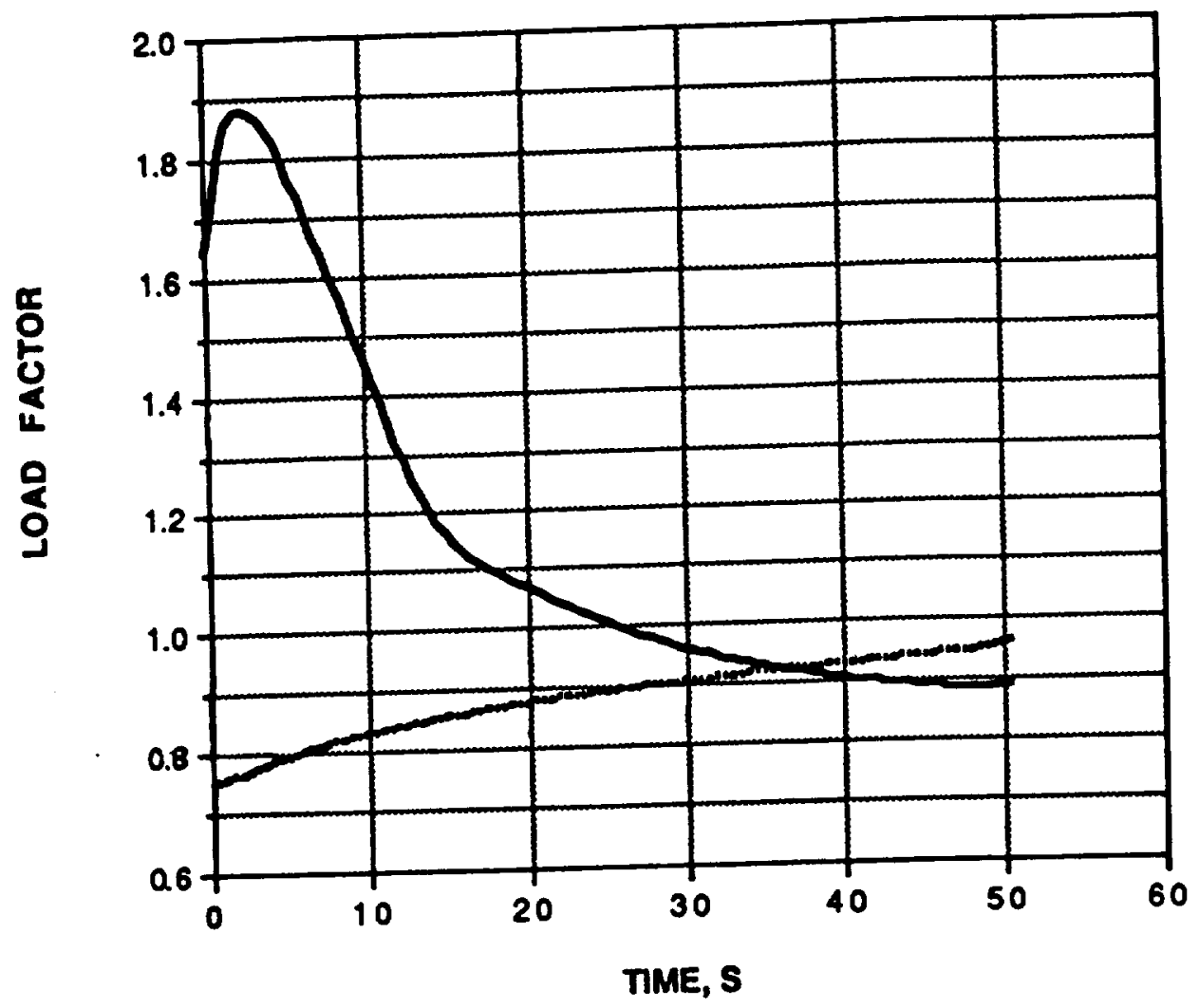

Fig. 6. Load Factor Histories for the Pursuer and the Evader. Dotted Line : Evader, Solld Line : Pursuer. 


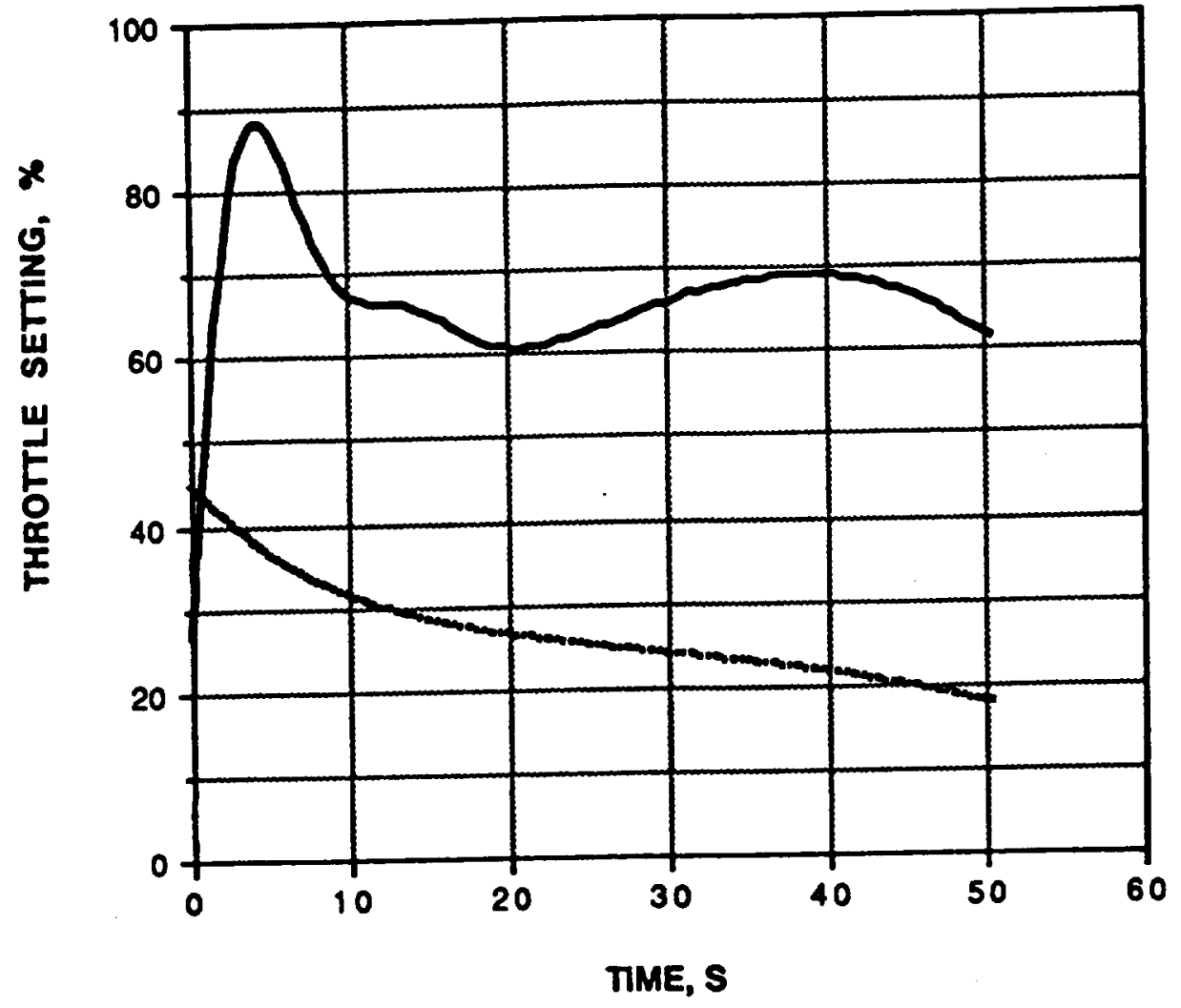

Fig. 7. Temporal Evolution of Pursuer-Evader Throttle Setting. Dotted Line : Evader, Solid Line : Pursuer. 


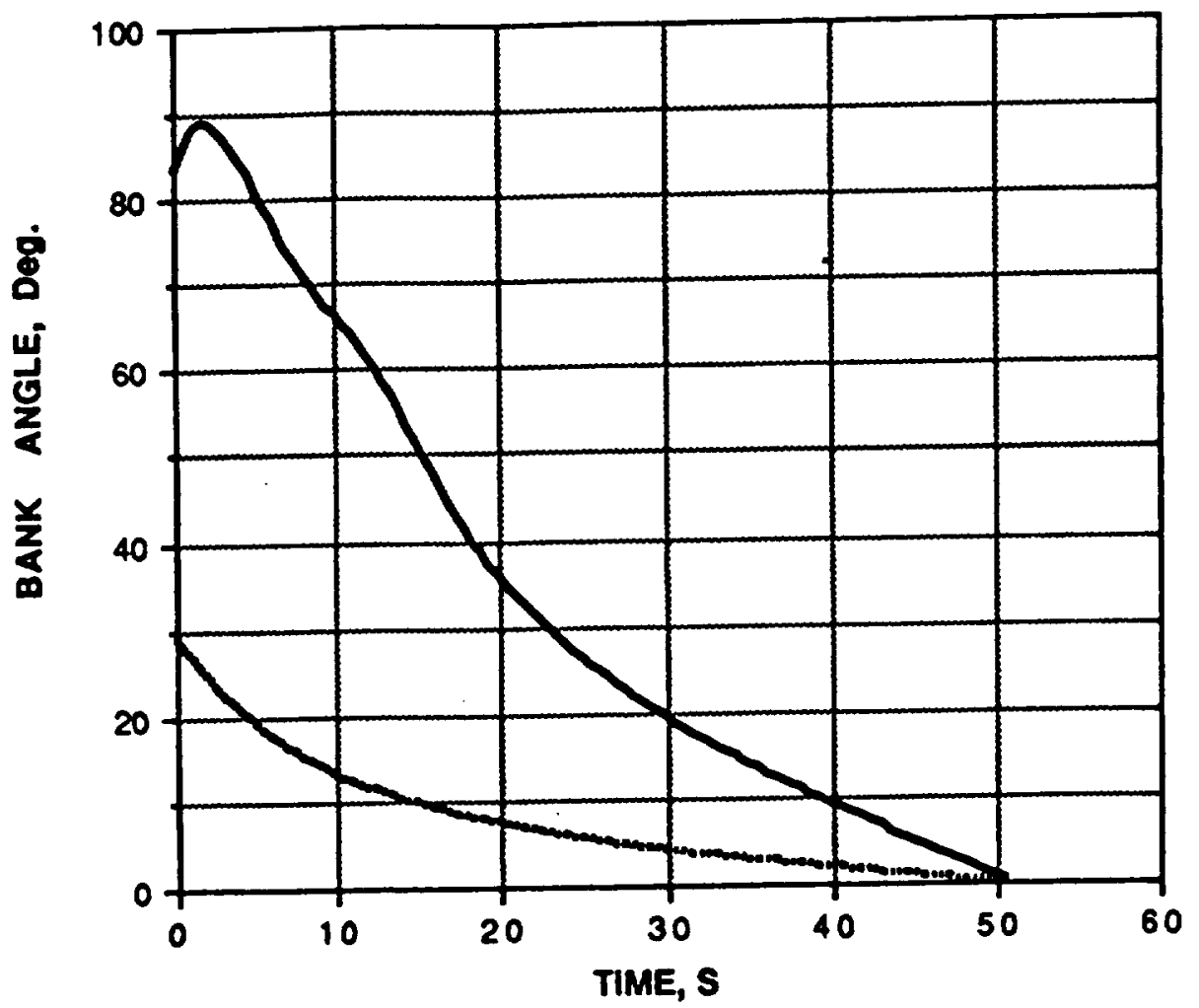

Fig. 8. Bank Angle Historles for the Pursuer and the Evader. Dotted Line : Evader, Solld Line : Pursuer. 


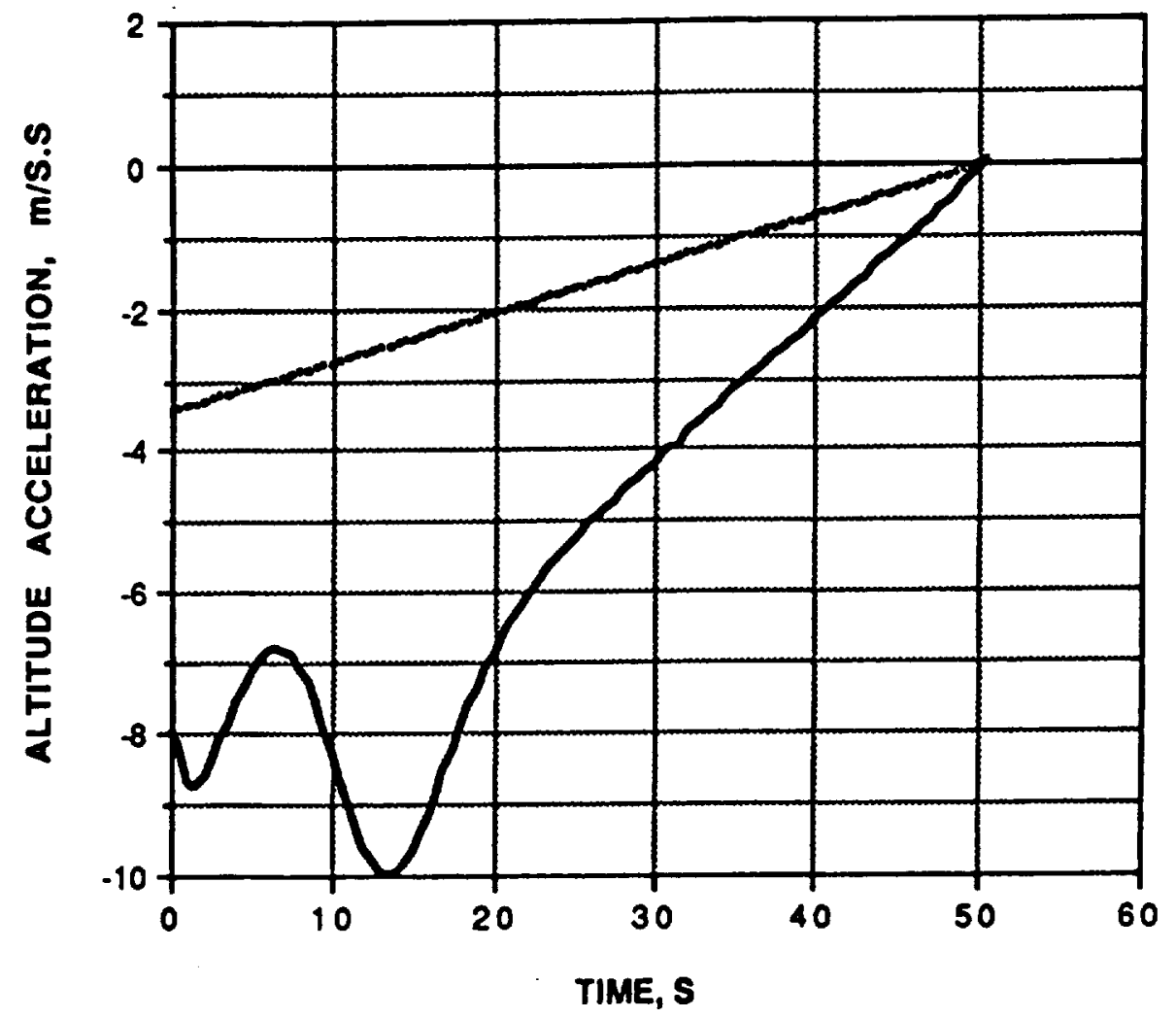

Fig. 9. Altitude Acceleration Historles for the Pursuer and the Evader. Dotted Line : Evader, Solld LIne : Pursuer. 